\title{
Adipokinetic hormone signalling system in the Chagas disease vector, Rhodnius prolixus
}

article version: post-print

Zandawala, Meet

Hamoudi, Zina

Lange, Angela B.

Orchard, Ian

\footnotetext{
"This is the peer reviewed version of the following article: Zandawala, M., Hamoudi, Z., Lange, A. B. and Orchard, I. (2015), Adipokinetic hormone signalling system in the Chagas disease vector, Rhodnius prolixus. Insect Mol Biol, 24: 264-276, which has been published in final form at doi:10.1111/imb.12157]. This article may be used for non-commercial purposes in accordance with Wiley Terms and Conditions for Self-Archiving."
}

\section{HOW TO CITE TSPACE ITEMS}

Always cite the published version, so the author(s) will receive recognition through services that track citation counts, e.g. Scopus. If you need to cite the page number of the TSpace version (original manuscript or accepted manuscript) because you cannot access the published version, then cite the TSpace version in addition to the published version using the permanent URI (handle) found on the record page. 


\section{Adipokinetic hormone signalling system in the Chagas disease vector, Rhodnius prolixus}

\author{
M. Zandawala, Z. Hamoudi, A. B. Lange and \\ I. Orchard \\ Department of Biology, University of Toronto \\ Mississauga, Mississauga, ON, Canada
}

\begin{abstract}
Neuropeptides and their G protein-coupled receptors are widespread throughout Metazoa and in several cases, clear orthologues can be identified in both protostomes and deuterostomes. One such neuropeptide is the insect adipokinetic hormone (AKH), which is related to the mammalian gonadotropinreleasing hormone. AKH has been studied extensively and is known to mobilize lipid, carbohydrates and proline for energy-consuming activities such as flight. In order to determine the possible roles for this signalling system in Rhodnius prolixus, we isolated the cDNA sequences encoding $R$.prolixus $A K H$ (Rhopr-AKH) and its receptor (Rhopr-AKHR). We also examined their spatial expression pattern using quantitative PCR. Our expression analysis indicates that $R$ hopr-AKH is only expressed in the corpus cardiacum of fifth-instars and adults. Rhopr-AKHR, by contrast, is expressed in several peripheral tissues including the fat body. The expression of the receptor in the fat body suggests that AKH is involved in lipid mobilization, which was confirmed by knockdown of Rhopr-AKHR via RNA interference. Adult males that had been injected with double-stranded RNA (dsRNA) for Rhopr-AKHR exhibited increased lipid content in the fat body and decreased lipid levels in the haemolymph. Moreover, injection of Rhopr-AKH in Rhopr-AKHR dsRNA-treated males failed to elevate haemolymph lipid levels, confirming that this is indeed the receptor for Rhopr-AKH.
\end{abstract}

\section{First published online 24 December 2014}

Correspondence: Meet Zandawala, Department of Biology, University of Toronto Mississauga, Mississauga, ON L5L 1C6, Canada. Tel.: + 1905828 5333; fax: + 1905828 3792; e-mail: meet.zandawala@ utoronto.ca
Keywords: insect, neuropeptide, gonadotropinreleasing hormone, G protein-coupled receptor, dsRNA.

\section{Introduction}

Adipokinetic hormone $(\mathrm{AKH})$ was the first insect neurohormone to be isolated, sequenced and synthesized (Stone et al., 1976; Broomfield \& Hardy, 1977) and is one of the most studied neuropeptides in insects. To date there are over 50 members of the now-termed $\mathrm{AKH}$ /red-pigment concentrating hormone (RPCH) family (Gäde \& Marco, 2013). First identified for their ability to mobilize lipid, carbohydrate or proline from the fat body during locomotory activities such as flight, AKHs have also been shown to have additional functions, such as stimulating heartbeat rate (Rosiński \& Gäde, 1988; Keeley et al., 1991; Noyes et al., 1995; Malik et al., 2012; Gäde \& Marco, 2013), inhibiting protein synthesis (Carlisle \& Loughton, 1986) and extending life span during starvation in Drosophila melanogaster (Isabel et al., 2005). Recently, AKH signalling has also been shown to play a role in oxidative stress (Bednarova et al., 2013).

It was over 25 years since the discovery of $\mathrm{AKH}$ that the first $A K H$ receptors (AKHRs) were identified in the fruit fly D. melanogaster and the silkworm Bombyx mori (Park et al., 2002; Staubli et al., 2002). These receptors are rhodopsin-like G protein-coupled receptors (GPCRs) and have been found to be structurally related to the vertebrate gonadotropin-releasing hormone $(\mathrm{GnRH})$ receptor in vertebrates. AKHRs were subsequently identified in Manduca sexta (Ziegler et al., 2011), Periplaneta americana (Hansen et al., 2006), Anopheles gambiae (Kaufmann \& Brown, 2006) and Aedes aegypti (Kaufmann et al., 2009), and many more have been deduced from genomic sequences of other insect species (see Grimmelikhuijzen \& Hauser, 2012; Hauser \& Grimmelikhuijzen, 2014). Owing to the growth in genomic data and availability of extensive amino acid sequences of GPCRs, Hauser \& Grimmelikhuijzen (2014) used an in silico approach to propose a scenario for the evolution of 
$\mathrm{AKH}$ and its receptor along with those of two other insect peptide signalling pathways, namely those of corazonin (CRZ) and AKH/CRZ-related peptide (ACP). In their scenario, these three ligands and their receptors originated from an ancestral $\mathrm{GnRH}$-like ligand and receptor, and duplication occurred before the emergence of Mollusca and Annelida, leading to two branches; one producing a CRZ-like receptor/ligand and the other an $\mathrm{AKH}$-like receptor/ligand. Further duplication of the AKH hormonal system led to the AKH and ACP systems that are present today in Arthropoda (see Roch et al., 2014). A recent study by Patel et al. (2014) examined these three peptide signalling pathways to determine if the co-evolution of these three peptide families with partially conserved amino acid sequences also resulted in shared physiological activities in the blood-gorging bug Rhodnius prolixus. Their study determined that Rhopr-AKH was capable of elevating haemolymph lipid levels in a dose-dependent manner in adult male insects (but had no effect on heartbeat frequency) whereas Rhopr-CRZ was capable of increasing heartbeat frequency in a dose-dependent manner (but had no effect on lipid mobilization). Rhopr-ACP had no effect on either haemolymph lipid levels or heartbeat frequency. ACP appears to have a widespread presence in insects and yet no physiological function has been identified in any insect. Although all three peptides share some amino acid sequence similarity, none of these peptides in An. gambiae cross-react with the receptors for the other ligands (Hansen et al., 2010). These data suggest that each peptide family has retained a distinct physiological function.

$A K H R$ transcript expression has been shown in the fat body, central nervous system (CNS) and ovaries of mosquitoes (Kaufmann \& Brown, 2006; Kaufmann et al., 2009). In P. americana, $A K H R$ is expressed in several tissues, including the brain, ovaries, flight muscles and digestive system (Wicher et al., 2006). Interestingly, the fact that AKHR is structurally related to the $\mathrm{GnRH}$ receptor in mammals, coupled with AKHR transcript expression in the fat body and ovaries, supports the notion that nutrient metabolism in females might be regulated to meet the demands not only for flight but also for reproduction. A role in reproduction in insects has yet to be proven, although there are several lines of indirect evidence. AKHR knockdown in the tsetse fly Glossina morsitans affects 'milk' production during tsetse pregnancy (Attardo et al., 2012) and AKH has been found to inhibit egg production indirectly in the cricket Gryllus bimaculatus (Lorenz, 2003). However, AKH has been shown to be involved in reproduction in the nematode Caenorhabditis elegans, with $\mathrm{AKH}-\mathrm{GnRH}$ knockdown leading to a delay in the timing of egg-laying and a decrease in the number of total progeny (Lindemans et al., 2009). Recent studies have shown that knockdown of $A K H R$ in $G$. bimaculatus using Grybi-AKHR doublestranded RNA (dsRNA) decreased levels of 1,2diacylglycerol and trehalose in the haemolymph whilst increasing the levels of triacylglycerol in the fat body (Konuma et al., 2012). This knockdown also enhanced starvation resistance, decreased locomotory activity and increased food intake in G. bimaculatus. This increase in food intake was the result of an increase in feeding frequency, indicating that $\mathrm{AKH}$ signalling is involved in nutritional control. Similar conclusions were obtained in experiments in which $\mathrm{AKH}$ injection or topical application activates locomotory and flight activity in orthopteran species, suggesting that $\mathrm{AKH}$ signalling might contribute to the regulation of feeding-related behaviours (Lorenz \& Anand, 2004; Wicher et al., 2006).

Previously, AKH has been sequenced in the kissing bug R. prolixus (Ons et al., 2011; Marco et al., 2013) and shown to be present in a group of neurosecretory cells in the corpus cardiacum (CC) (Patel et al., 2014). Injection of Rhopr-AKH into adult males elevated haemolymph lipid levels in a dose-dependent manner (Patel et al., 2014). Haemolymph lipid levels also increase during flight in $R$. prolixus and this elevation in lipid levels may be the result of the release of Rhopr-AKH (Ward et al., 1982). Rhopr-AKH does not alter heartbeat rate in $R$. prolixus although it has been shown to alter heart rate frequency in other insects (Rosiński \& Gäde, 1988; Keeley et al., 1991; Noyes et al., 1995; Malik et al., 2012). In the present study, the cDNA sequences were cloned for Rhopr-AKH and Rhopr-AKHR and quantitative PCR (qPCR) was used to determine their spatial expression profiles. As expected, Rhopr-AKH was only expressed in the CC and Rhopr-AKHR was enriched in the fat body as well as in adult reproductive tissues. RNA interference (RNAi) experiments were performed to knockdown Rhopr-AKHR and the effect on lipid mobilization from the fat body was examined.

\section{Results}

Rhopr-AKH and Rhopr-AKHR

First, we cloned and sequenced the complete cDNA encoding Rhopr-AKH (Fig. 1A). The sequence is at least $393 \mathrm{bp}$ long and includes a 216-bp open reading frame (ORF). The $5^{\prime}$ and $3^{\prime}$ untranslated regions (UTRs) are at least 108 and $69 \mathrm{bp}$ long, respectively. There is an in-frame stop codon upstream of the start codon, confirming that the ORF is complete. Within the prepropeptide sequence, a signal peptide cleavage site is present between the alanine residue at position 21 and glutamine at position 22. The mature peptide is flanked by the signal peptide at the $\mathrm{N}$-terminus and a lysine and arginine dibasic cleavage site at the $\mathrm{C}$-terminus. Molecular organization of Rhopr-AKH shows that the gene comprises three 
A

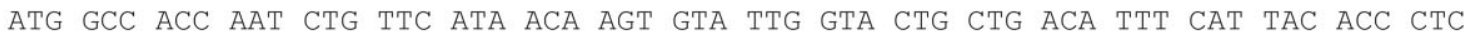

MET Ala Thr Asn Leu Phe Ile Thr ser Val Leu Val Leu Leu Thr Phe His Tyr Thr Leu

GCA CAG CTA ACA TTT TCA ACC GAC TGG GGT AAG AGA TCA GTT CGC CAC AAT GCA CCT GAT

Ala Gln Leu Thr Phe Ser Thr Asp Trp Gly Lys Arg Ser Val Arg His Asn Ala Pro Asp

TGT ACA CCA AAT CCT GAT ACt GTA ATT TTT CTG TAC AAg tAT TTG CAg AAt GAg tTT TAC

Cys Thr Pro Asn Pro Asp Thr Val Ile Phe Leu Tyr Lys Tyr Leu Gln Asn Glu Phe Tyr

B

Rhopr-AKH

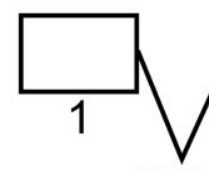

719 bp

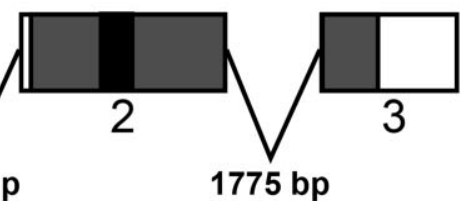

1775 bp

$\square$ Untranslated region

Precursor peptide

\section{$\longmapsto 100$ bp}

Mature peptide

C
Rhopr
Nillu
Drome
Trica
MAT-NLFITSVLVLLTFHYTLAQLTFSTDWGKRSVRH
-MARAIAVLICALIMAVICTAQVNFSPNWGKRAASS
MNPKSEVLIAAVLFMILACVQCQLTFSPDWGKRSVGG
--MSRM-FLIVVLIAFVGVCTAQLNFSTDWGKRSGSS
HEN---
APDCTPNPDTVIF LYYIQNEFYKMIFCGKTTEGL 71 MNPKSEVLIAAVLFMLIACVOCOLTFSPDWGKRSVGGAFPGTFFET-QQGNCKTSN MLIEIFRFV SQAQLFLDCKHRE-- 79
Anoga MDTVKLFTVLICASIMLITEAQLTETPAWGKRSQGAMG Signal peptide
Mature peptide

Figure 1. Rhodnius prolixus adipokinetic hormone (Rhopr-AKH) sequence and structure. (A) Rhopr-AKH cDNA sequence and the deduced amino acid sequence. The numbering for each sequence is shown on the right. Within the nucleotide sequence, the exon-exon boundaries are shaded in grey and an in-frame stop codon upstream of the start codon is double underlined. Within the amino acid sequence, the start codon has been capitalized, the mature peptide is highlighted in black, the predicted site for signal peptide cleavage is marked by an arrow and the dibasic cleavage site has been underlined. (B) Rhopr-AKH gene structure. The boxes represent exons that have been drawn to scale. (C) Multiple sequence alignment of select AKH prepropeptides. Rhopr-AKH prepropeptide was aligned with its orthologues from Nilaparvata lugens (Nillu), Drosophila melanogaster (Drome), Tribolium castaneum (Trica) and Anopheles gambiae (Anoga). Identical amino acids have been highlighted in black and similar amino acids in grey if at least $50 \%$ of the sequences are similar.

exons, which are 100, 176 and 117 bp long (Fig. 1B). Two small introns (719 and $1775 \mathrm{bp}$ ) separate these exons. The mature peptide is encoded by exon 2 and is very well conserved across other insects (Fig. 1C).

Next, we isolated the complete cDNA sequence encoding Rhopr-AKHR (Fig. 2A). This sequence is $1384 \mathrm{bp}$ long. The ORF is $1062 \mathrm{bp}$ long and encodes a receptor comprising of 353 amino acids. The $5^{\prime}$ and $3^{\prime}$ UTRs are at least 101 and $221 \mathrm{bp}$ long, respectively. The 5' UTR also contains an in-frame stop codon upstream of the start codon, confirming that this ORF is also complete. RhoprAKHR has all the characteristics of a GPCR: an extracellular $\mathrm{N}$-terminus, seven transmembrane domains and an intracellular C-terminus. It is also predicted to contain one $\mathrm{N}$-linked glycosylation site in its $\mathrm{N}$-terminus and 10 phosphorylation sites. Moreover, Rhopr-AKHR is highly predicted ( $99 \%$ probability) to couple with the $G$ stimulatory/G other alpha subunit by two different algorithms. PRED-COUPLE 2.0 also predicted the receptor to couple with the $\mathrm{Gq} / 11$ alpha subunit, although with a much 
A

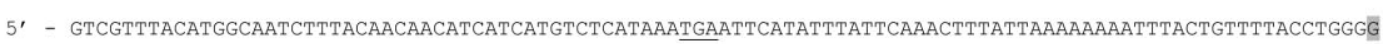

ATG ACA AGA ACG GAA GAA GTA TTT TCC TTT ACA TTT TTT CCG GAA TGG TCA GAg ATC AGA ACC GAA AAA AAT GAA ACA TAC GTT ATA CCT MET Thr Arg Thr Glu Glu Val Phe Ser Phe Thr Phe Phe Pro Glu Trp Ser Glu Ile Arg Thr Glu Lys Asn Glu Thr Tyr Val Ile Pro CCC GAC ATG AGA TTT AAC GAA GGA CAC AAG CTG GCT TTA GCA GTA TAT TCC TTA CTG ATG TTG ATA TCT GGA GTG GGC AAT GTG TGG GTG Pro Asp Met Arg Phe Asn Glu Gly His Lys Leu Ala Leu Ala Val Tyr Ser Leu Leu Met Leu Ile Ser Gly Val Gly Asn Val Trp Val TTG GTG AGA CTG GCC AAA TCA AGG AGA TCT CGC ACT AAT AGG ATG TTG ACG CAT CTG GCT ATC GCT GAC CTG TTT GTG GCC TTT CTC ATG Leu Val Arg Leu Ala Lys Ser Arg Arg Ser Arg Thr Asn Arg Met Leu Thr His Leu Ala Ile Ala Asp Leu phe Val Ala Phe Leu Met ATG CCT GCA GAG ATA CTT TCG GCG GCA ACA GTC GCC TGG TGG TTT GGA GAT ATA CCA TGT CGG ATA TTT GCA TTT TTC AAG ACT TTC GGA Met Pro Ala Glu Ile Leu Ser Ala Ala Thr Val Ala Trp Trp Phe Gly Asp Ile Pro Cys Arg Ile Phe Ala Phe Phe Lys Thr Phe Gly CTA TAC CAA AGC AGT TTT GTT TTG GTT TGC ATT GGA ATT GAT AGA TAT TAT GCA ATA GTT AAA CCA CTG AGC ATC AAG GAT ACA TAT TGC Leu Tyr Gln Ser Ser phe Val Leu Val Cys Ile Gly Ile Asp Arg Tyr Tyr Ala Ile Val Lys Pro Leu Ser Ile Lys Asp Thr Tyr Cys CGA GGA AAA GGC ATT GTA GCG TTA GCC TGG GTG ATT TCC GGC ATT TGC AGC TTG CCA CAA GTG GTT GTG TTC AGA GAA CAA GAG CAT TAC Arg Gly Lys Gly Ile Val Ala Leu Ala Trp Val Ile Ser Gly Ile Cys Ser Leu Pro Gln Val Val Val Phe Arg Glu Gln Glu His Tyr ACT TTC ACT GGG TAT AAA CAA TGT TGT ACA TTC AAT GCG TTT CCC ACG TCG TCA CAC GAA ATA GCA TAT AGC ATG TAT AAC ATG GCA ATG Thr Phe Thr Gly Tyr Lys Gln Cys Cys Thr Phe Asn Ala Phe Pro Thr Ser Ser His Glu Ile Ala Tyr Ser Met Tyr Asn Met Ala Met ATG TAC ATG CTG CCA TTA GTG GTC ATC ATC TTC TGT TAT GGC TCC ATC TTC ATA GAA ATA TAT CGC AGG ACT TCA GCT CAA AAC TCA GGC Het Tr Met Leu Pro Leu Val Val Ile Ile Phe Cys Tyr Gly Ser Ile Phe Ile Glu Ile Tyr Arg Arg Thr Ber Ala Gln Asn Ser Gly Lys Leu Arg Arg Ser Thr Leu Gly Phe Leu Gly Arg Ala Lys Asn Arg Thr Leu Lys Leu Thr Ile Thr Ile Ile Leu Ala Phe Phe Ile TGC TGG ACA CCT TAT ATC ATG GCT TTA TGG TAC TGG CTC GAC CGC AGC ACG GCA GAA GGT GTG GAT GTG CGC GTA AAA AGA GCT CTA Cys Trp Thr Pro Tyr Tyr Ile Met Ala Leu Trp Tyr Trp Leu Asp Arg Ser Thr Ala Glu Gly Val Asp Val Arg Val Lys Arg Ala Leu TTT CTA TTC GCA TGC ACC AAC TCT AGT ATT AAC CCA CTC GTC TAT GGT GTC TAC CAG CGT ACA GGC TGT GGG CCA AAC AAC TCG CGC ACT Phe Leu Phe Ala Cys Thr Asn Ser Ser Ile Asn Pro Leu Val Tyr Gly Val Tyr Gln Arg Thr Gly Cys Gly Pro Asn Asn Ser Arg Thr

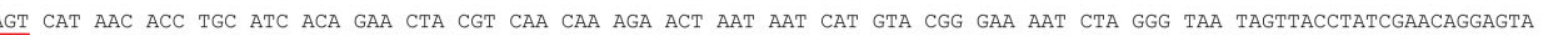
Ir His Asn Thr Cys Ile Thr Glu Leu Arg Gln Gln Arg Thr Asn Asn His Val Arg Glu Asn Leu Gly *

TTCGTGTTCAAGGATTATCCAAAAATAAGTGCAACAAGTGTCACAGCTAAAGCAAAGTGTGTAAAATATTTTTTTCTTTTATCGTCCAAAGCCATTTGTATAGATTAGTTGCAAATGGG CGTATCGTATCACCGTTGCAGTTAAAGAAGTTTTTATAAAATCATGTTTTCACAGGAATTACTTGTCTTGAGGTCTAGT - $3^{\prime}$

B

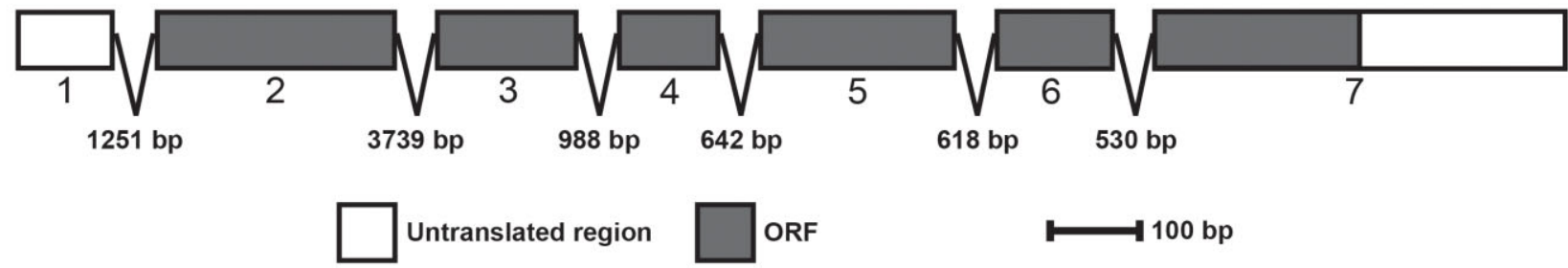

Figure 2. Rhodnius prolixus adipokinetic hormone receptor (Rhopr-AKHR) sequence and structure. (A) Rhopr-AKHR cDNA sequence and the deduced amino acid sequence. The numbering for each sequence is shown on the right. Within the nucleotide sequence, the exon-exon boundaries are shaded in grey. Within the amino acid sequence, the initial methionine start codon has been capitalized, the seven predicted transmembrane domains are highlighted in black, one predicted $\mathrm{N}$-linked glycosylation site has been boxed and 10 predicted phosphorylation sites have been highlighted in red. (B) Rhopr-AKHR gene structure. The boxes represent exons that have been drawn to scale. ORF, open reading frame.

lower probability (53\%). Lastly, Rhopr-AKHR comprises seven exons and the ORF spans exons 2 to 7 (Fig. 2B).

\section{Sequence and phylogenetic analysis}

We aligned Rhopr-AKHR with other insect AKHRs and Homo sapiens $\mathrm{GnRH}-\mathrm{R} 1$ to examine the conservation across various species (Fig. 3). The alignment shows that the region between the seven transmembrane domains is well conserved; however, the $\mathrm{N}$ - and $\mathrm{C}$-termini are less conserved. The putative $\mathrm{N}$-linked glycosylation site in the $\mathrm{N}$-terminus is also conserved across all of the receptors examined. Moreover, several functionally important residues in $H$. sapiens $\mathrm{GnRH}-\mathrm{R} 1$ are also conserved in Rhopr-AKHR and other orthologues. These include residues that are involved in receptor activation, binding pocket formation, ligand binding, protein kinase $\mathrm{C}$ phosphorylation, G stimulatory alpha coupling and $\mathrm{Gq} / 11$ alpha coupling (see Millar et al., 2004).

Phylogenetic analysis of $\mathrm{AKH}, \mathrm{ACP}, \mathrm{CRZ}$ and $\mathrm{GnRH}$ receptors confirmed the evolutionary relatedness amongst these hormonal systems (Fig. 4). Hence, AKHRs and ACPRs in Protostomia are sister to a clade of GnRHRs in Deuterostomia that includes human GnRH-R1 and amphioxus (Branchiostoma floridae) GnRH-R1 and R2. However, CRZRs are sister to $\mathrm{Br}$. floridae $\mathrm{GnRH}-\mathrm{R} 3$ and R4. Rhopr-AKHR is closely related to other insect receptors that have previously been functionally characterized as AKHR. These include receptors from D. melanogaster (Staubli et al., 2002) and An. gambiae (Hansen et al., 2010), thus supporting the claim that the receptor isolated from $R$. prolixus is an AKHR. 


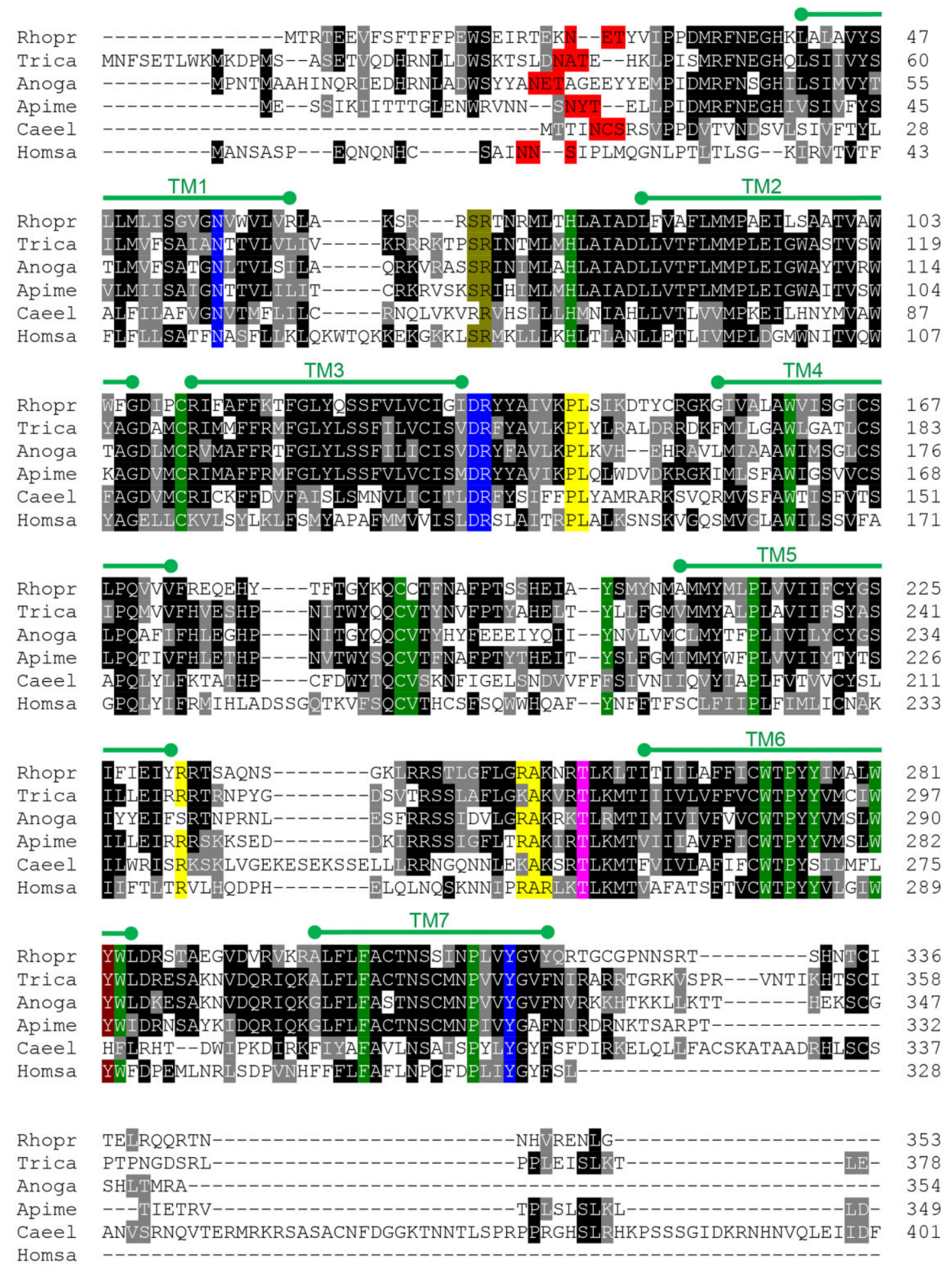

Figure 3. Multiple sequence alignment of select invertebrate adipokinetic hormone (AKH) receptors and Homo sapiens gonadotropin-releasing hormone receptor 1 (GnRH-R1). Rhodnius prolixus AKH receptor (Rhopr-AKHR) was aligned with its orthologues from Tribolium castaneum (Trica), Anopheles gambiae (Anoga), Apis mellifera (Apime), Caenorhabditis elegans (Cael) and H. sapiens (Homsa). Identical amino acids have been highlighted in black and similar amino acids in grey if at least $50 \%$ of the sequences are similar. The predicted locations of the seven transmembrane domains of Rhopr-AKHR have been indicated using green lines and the predicted N-linked glycosylation site has been highlighted in red. Functionally important amino acid residues in human $\mathrm{GnRH}-\mathrm{R} 1$ and their putative homologues in the other sequences have been highlighted using the following scheme: residues important in receptor activation (blue), binding pocket formation (green), ligand binding (dark red), protein kinase C (PKC) phosphorylation (pink), Gs alpha coupling (olive green) and Gq/11 alpha coupling (yellow) (see Millar et al., 2004). 


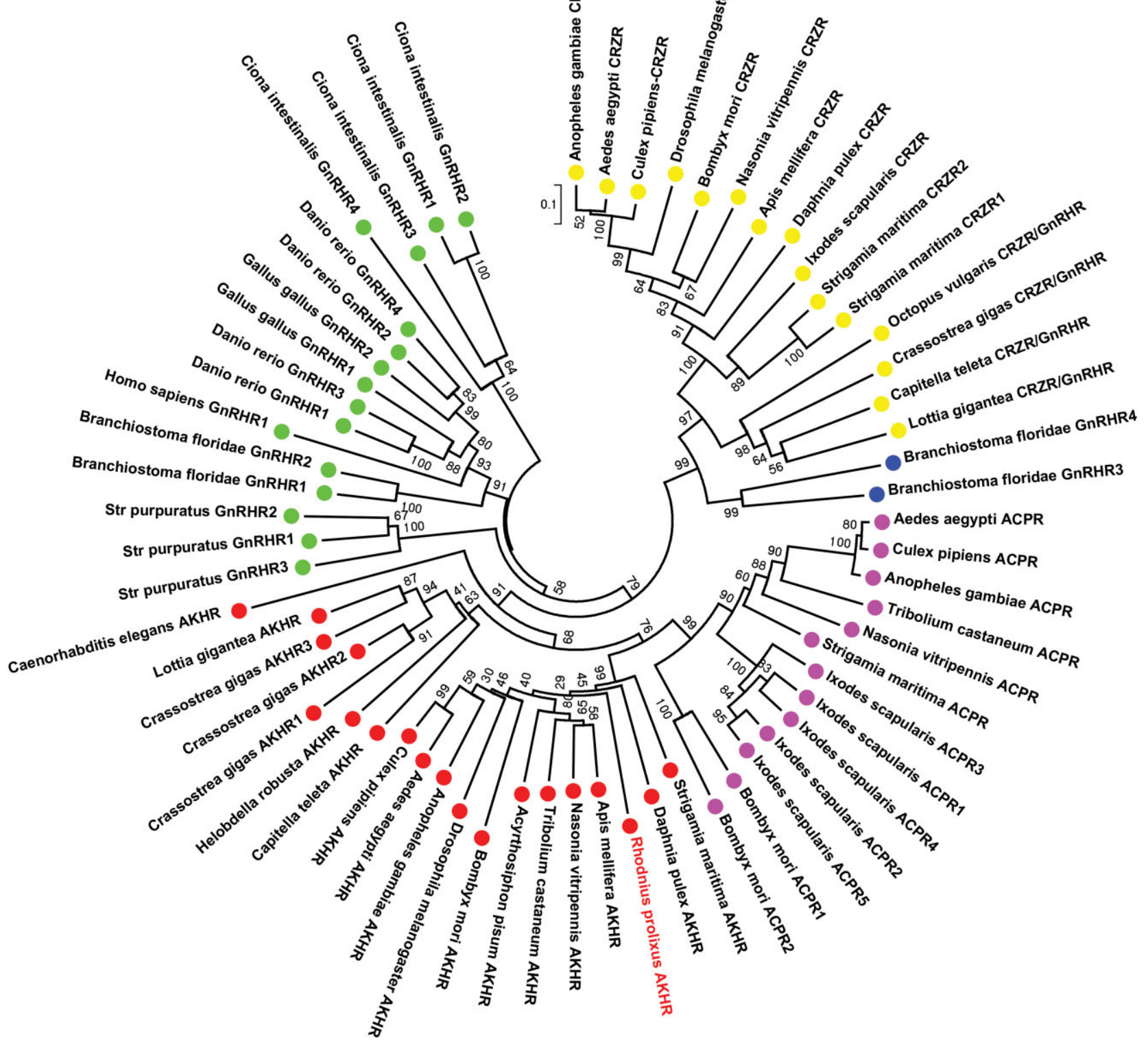

Figure 4. A phylogram of adipokinetic hormone (AKH), AKH/corazonin (CRZ)-related peptide (ACP), CRZ and gonadotropin-releasing hormone (GnRH) receptors obtained following a neighbour-joining analysis (1000 bootstrap replicates). The taxa are labelled using species names. Str purpuratus refers to Strongylocentrotus purpuratus. Note that the AKH receptors in protostomes are sister to the GnRH receptors in deuterostomes, which include human GnRH-R1 and amphioxus (Branchiostoma floridae) GnRH-R1 and R2.

\section{Spatial expression profile of Rhopr-AKH and Rhopr-AKHR}

We examined Rhopr-AKH expression in the CNS and the CC/corpora allata (CA) complex of both fifth-instars and adults using qPCR. Our analysis indicates that Rhopr$A K H$ is expressed in the CC/CA complex but not in the CNS of either fifth-instars or adults (Fig. 5A). Moreover, the expression in fifth-instars is almost twofold higher than that seen in adults. By contrast, Rhopr-AKHR is expressed in both the CNS and the CC/CA complex of fifth-instars and adults (Fig. 5B).

Spatial expression profiling of Rhopr-AKHR was performed via qPCR to identify possible target tissues of Rhopr-AKH. Within the fifth-instar, Rhopr-AKHR was highly expressed in a pool of tissues comprising the fat bodies, diaphragm and abdominal nerves, and also in prothoracic glands and associated fat bodies (Fig. 6). The 
A

Figure 5. Spatial expression analysis of (A) Rhodnius prolixus adipokinetic hormone (Rhopr-AKH) and (B) Rhopr-AKH receptor (Rhopr-AKHR) in the $R$. prolixus central nervous system (CNS) determined using quantitative PCR. Expression was analysed in the following tissues: fifth-instar CNS (5th CNS), fifth-instar corpora cardiaca and corpora allata (5th CC/CA), adult CNS (Ad. CNS) and adult corpora cardiaca and corpora allata (Ad. CC/CA). Expression is shown relative to transcript levels in fifth instar CC/CA cDNA $(n=3)$. Data are mean \pm SEM.

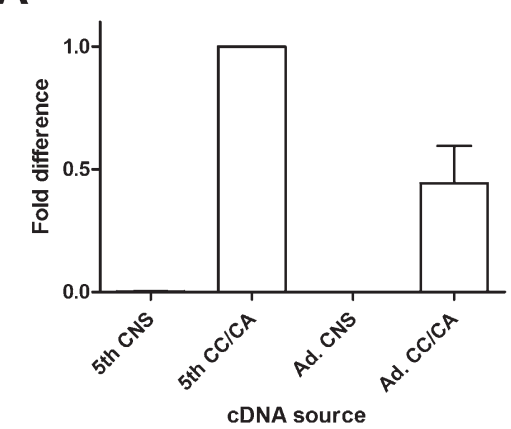

B

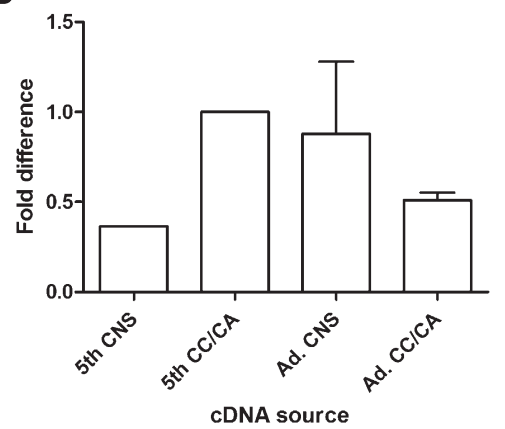

transcript was expressed at moderate levels in the dorsal vessel and at lower levels in the CNS and female reproductive tissues (Fig. 6). Within the adult reproductive tissues, the highest expression for Rhopr-AKHR was observed in testes and the second highest abundance in a pool of female reproductive tissues comprising the bursa, oviducts, spermatheca and cement gland (Fig. 7). The receptor was also expressed, albeit at lower levels, in the ovaries and a pool of male reproductive tissues comprising the vas deferens, seminal vesicle, accessory glands and ejaculatory duct.

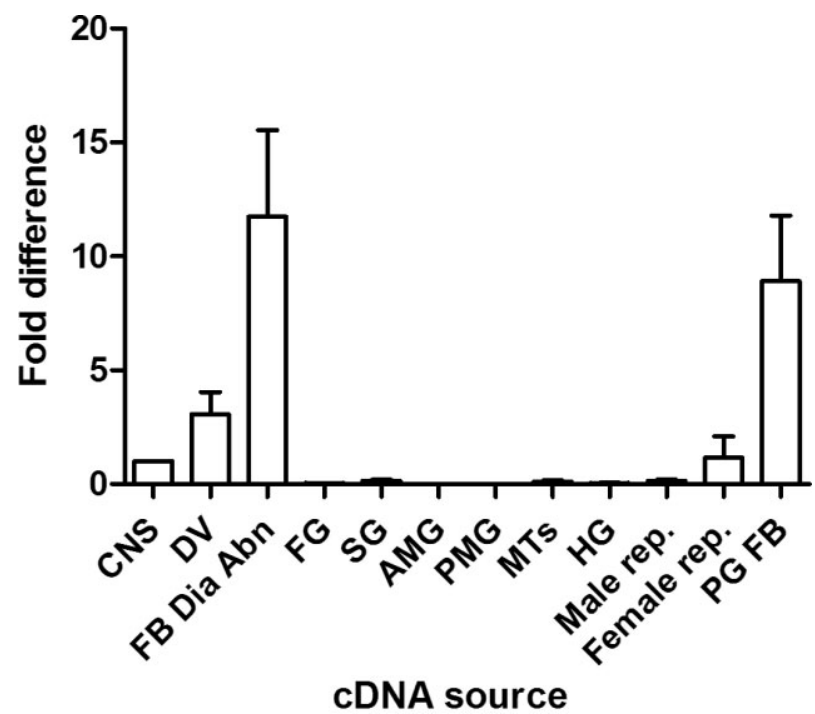

Figure 6. Spatial expression analysis of Rhodnius prolixus adipokinetic hormone receptor (Rhopr-AKHR) in fifth instar $R$. prolixus determined using quantitative PCR. Expression was analysed in the following tissues: central nervous system (CNS), dorsal vessel (DV), fat bodies, diaphragm and abdominal nerves (FB Dia Abn), foregut (FG), salivary glands (SG), anterior midgut (AMG), posterior midgut (PMG), Malpighian tubules (MTs), hindgut (HG), male reproductive tissues (Male rep.),. female reproductive tissues (Female rep) and prothoracic glands and associated fat body (PG FB). Expression is shown relative to transcript levels in CNS cDNA $(n=2)$. Data are mean \pm SEM.

\section{Rhopr-AKHR in lipid mobilization}

Adult male $R$. prolixus were injected 10 days after bloodgorging with $2 \mu \mathrm{g}$ of either ampicillin-resistance gene (ARG) or Rhopr-AKHR dsRNA. Rhopr-AKHR dsRNAinjected insects exhibited a greater than $97 \%$ knockdown 4 days post-injection (Fig. 8). Four days post-injection of Rhopr-AKHR dsRNA, ie 14 days post blood-gorging, the haemolymph lipid levels were significantly reduced (Fig. 9A) and the ventral abdominal fat body lipid content was significantly increased compared with controls (Fig. 9B).

To verify the importance of Rhopr-AKHR in lipid mobilization from the fat body, we investigated if Rhopr-AKH was still capable of mobilizing lipid in adult male $R$. prolixus after knocking down the Rhopr-AKHR using dsRNA (Fig. 10). Rhopr-AKH (5 pmol) injected into

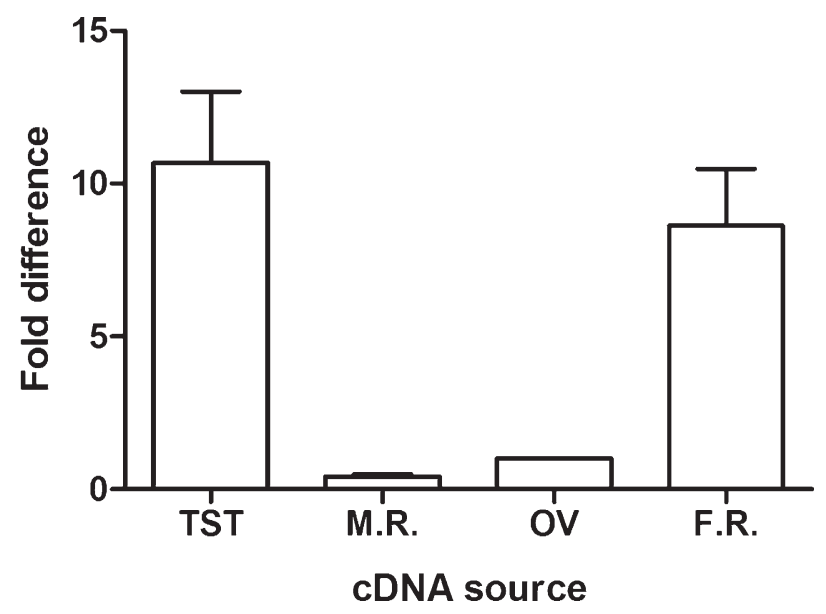

Figure 7. Spatial expression analysis of Rhodnius prolixus adipokinetic hormone receptor (Rhopr-AKHR) in $R$. prolixus adult reproductive tissues determined using quantitative PCR. Expression was analysed in the following tissues: testes (TST), rest of the male reproductive tissues (M.R.), ovaries (OV) and rest of the female reproductive tissues (F.R). Expression is shown relative to transcript levels cDNA of ovaries $(n=3)$. Data are mean \pm SEM. 


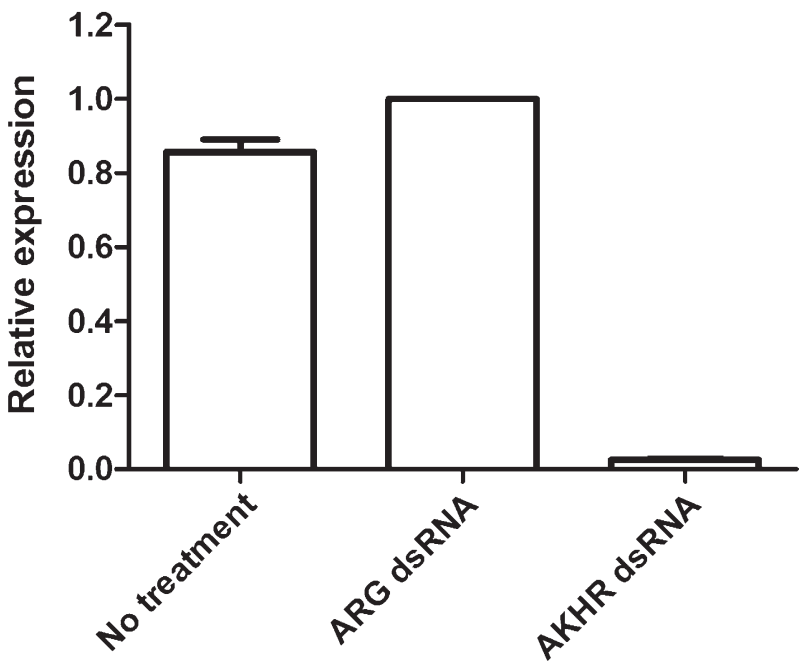

Figure 8. Effect of double-stranded RNA (dsRNA) injection on Rhodnius prolixus adipokinetic hormone receptor (Rhopr-AKHR) transcript levels in adult male ventral abdominal fat body. Adult males were left untreated, or injected with either ampicillin-resistance gene (ARG) dsRNA or Rhopr-AKHR dsRNA. Transcript levels were determined in fat body 4 days post-injection. Results are presented as means of three independent biological replicates. Rhopr-AKHR dsRNA-injected insects exhibited $>97 \%$ knockdown compared with ARG dsRNA injected insects $(n=3)$. Data are mean \pm SEM.

$R$. prolixus that had received no treatment (NT) led to a significant increase in haemolymph lipid levels as it did in those insects previously injected with ARG dsRNA (Fig. 10). Insects injected with Rhopr-AKHR dsRNA did not significantly increase their haemolymph lipid levels in response to Rhopr-AKH injection (Fig. 10).

\section{Discussion}

In the present study, we isolated and characterized cDNA sequences encoding $\mathrm{AKH}$ and $\mathrm{AKHR}$ from the Chagas

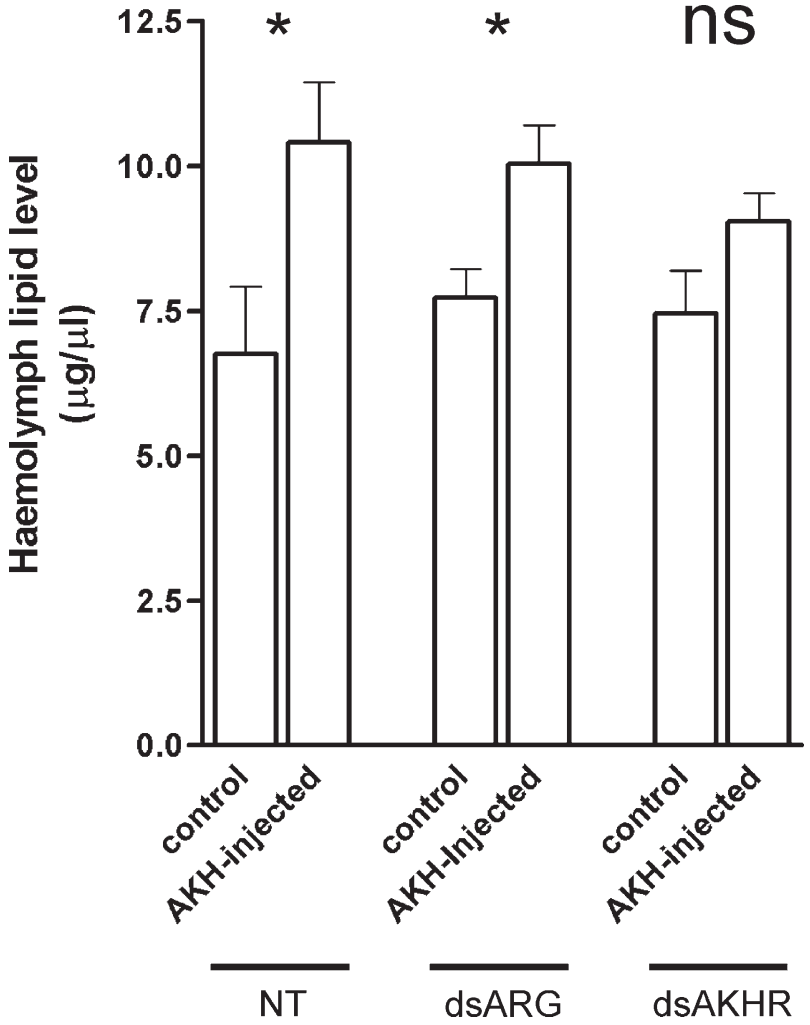

Figure 10. Functional characterization of Rhodnius prolixus adipokinetic hormone receptor (Rhopr-AKHR) using RNA interference. Changes in haemolymph lipid levels following injection of saline (control) or Rhopr-AKH (AKH-injected). Knockdown of Rhopr-AKHR with AKHR double-stranded RNA (dsAKHR) resulted in a reduction in the increase in haemolymph lipid levels 90 min after injection of 5 pmol Rhopr-AKH compared with no treatment (NT) insects [not treated with double-stranded RNA (dsRNA)] and insects injected with ampicillin-resistance gene (ARG) dsRNA (dsARG). Data were analysed using a one-way ANOVA $(P=0.063)$ followed by Bonferroni's multiple comparison test ( ${ }^{*}, P<0.05$; ns, not significant). Data are mean \pm SEM ( $n=7$ for NT; $n=14-16$ for ARG dsRNA and AKHR dsRNA).
A

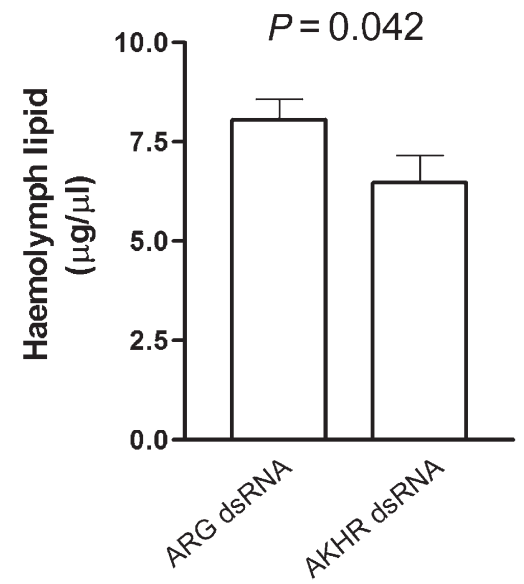

B

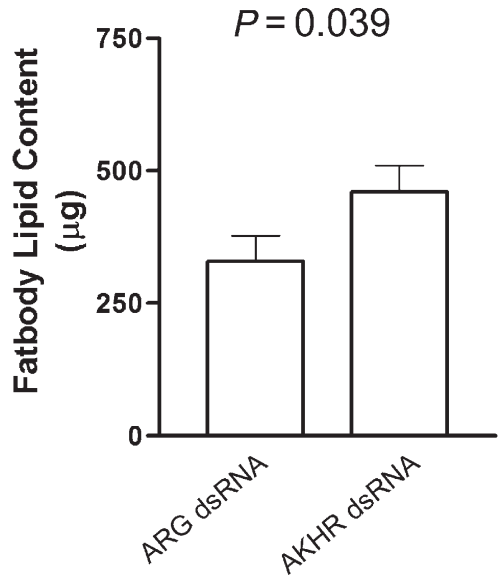

Figure 9. Effect of Rhodnius prolixus adipokinetic hormone receptor (Rhopr-AKHR) knockdown on (A) haemolymph lipid levels and (B) fat body lipid content of adult males. Rhopr-AKHR knockdown caused a decrease in haemolymph lipid levels and an increase in fat body lipid content compared with ampicillin-resistance gene (ARG) double-stranded RNA-injected insects. Statistical analysis was performed using a one-tailed $t$-test $(n=11-13)$. Data are mean \pm SEM. 
disease vector, $R$.prolixus (Rhopr-AKH and Rhopr$A K H-R$, respectively). Two sequences for Rhopr-AKH are found in GenBank. These sequences are $291 \mathrm{bp}$ (GU062794.1) and $320 \mathrm{bp}$ long (GQ888667.1) and encode prepropeptides comprising 96 amino acids and 71 amino acids, respectively. In order to clarify this discrepancy, we cloned and sequenced the complete cDNA encoding Rhopr-AKH. The sequence presented here is more similar to the sequence under the accession number GQ888667.1. The ORF presented here differs by only one nucleotide (an adenine at position 314 in place of guanosine). This also alters the amino acid from glutamate to glycine. The sequence presented here also has the additional $5^{\prime}$ UTR. This sequence verification and extension was essential as it allowed for the design of suitable primers to perform qPCR and thereby examine the expression pattern of Rhopr-AKH. A previous report examining the distribution of $\mathrm{AKH}$ in $R$. prolixus using immunohistochemistry was unable to conclusively determine the expression pattern as a result of the anti-AKH antiserum possibly cross-reacting with an ACP-like substance (Patel et al., 2014). Hence, in order to clarify these data, we examined the expression of Rhopr-AKH in CNS and the CC/CA complex. Not surprisingly, Rhopr-AKH was expressed in the CC/CA complex but not in the CNS. Interestingly, the expression in fifth-instar CC/CA was almost twofold higher than that seen in adult CC/CA. The significance of this difference is still unclear as one might have predicted higher $A K H$ expression (and the subsequent $\mathrm{AKH}$ production) in adults because of energy demands associated with flight and reproduction. This study shows that in the CNS, $A K H$ is solely expressed in the neurosecretory cells of the $R$. prolixus $\mathrm{CC}$ as has been shown for several other insects (see Schooley et al., 2012; Audsley et al., 2014). Nonetheless, there are a few studies that have reported the presence of $\mathrm{AKH}$-like material in insect brains via immunohistochemical or mass spectrometric analyses (Siegert, 1999; Kaufmann \& Brown, 2006; Kaufmann et al., 2009). With the recent discovery of the ACP signalling system, it is now evident that this is ACP and not AKH (Hansen et al., 2010). Caution must therefore be exercised when interpreting results of studies prior to the discovery of the ACP signalling system.

Several lines of evidence confirm that the putative Rhopr-AKHR isolated here is indeed an AKHR. Firstly, several functionally important residues that are involved in receptor activation, binding pocket formation and ligand binding in $H$. sapiens $\mathrm{GnRH}-\mathrm{R} 1$ are also conserved in Rhopr-AKHR (Millar et al., 2004). Secondly, our phylogenetic analysis shows that the receptor is orthologous to other insect receptors that have been functionally characterized as AKHR. Lastly, and most importantly, Rhopr-AKH injection failed to significantly increase the haemolymph lipid levels in insects in which the receptor was knocked down via RNAi. Furthermore, insects with Rhopr-AKHR knocked down exhibited reduced haemolymph lipid levels and increased fat body lipid content in comparison with control insects. Similar results have been obtained in G. bimaculatus wherein knockdown of $A K H R$ resulted in decreased haemolymph 1,2 diacylglycerol and trehalose levels and an increase in triacylglycerol in the fat body (Konuma et al., 2012). Clearly, the $\mathrm{AKH}$ signalling pathway is important for maintaining metabolic homeostasis.

Spatial expression analysis of Rhopr-AKHR demonstrated that it is highly expressed in fat bodies of fifthinstar $R$. prolixus. This is not surprising as Rhopr-AKH has previously been shown to mobilize lipids, most probably from fat bodies in R. prolixus (Marco et al., 2013; Patel et al., 2014). Rhopr-AKHR transcript was also highly expressed in the sample comprising prothoracic glands (PGs) and associated fat bodies. It can be assumed that Rhopr-AKHR has little to no expression in $\mathrm{PGs}$ because preliminary results reveal that Rhopr-AKH has no effect on ecdysteroid synthesis by PGs (X. Vafopoulou, pers. comm.). Thus, most of the expression in the sample in the present study can probably be attributed to the fat bodies. Rhopr-AKHR is also moderately expressed in the dorsal vessel; this is intriguing because although Rhopr-AKH does not alter heartbeat frequency (Patel et al., 2014), it may increase the flow of haemolymph by modulating the stroke volume without affecting the heart rate via a Frank-Starling-like mechanism (da Silva et al., 2011). Rhopr-AKHR is expressed at low levels in the CNS and female reproductive tissues. Rhopr-AKHR transcript expression was observed in both the CNS and the isolated CC/CA complex of fifth-instars and adults. Hence Rhopr-AKH, which is produced by the $\mathrm{CC}$, may also act on the CC/CA complex to affect the synthesis and/or release of other hormones. Under this scenario, Rhopr-AKH would act as a releasing hormone, and thus be functionally conserved with $\mathrm{GnRH}$. With regards to the receptor expression in the CNS, AKH has been shown to cross the ganglionic sheath in P. americana (Wicher et al., 2006). Thus, Rhopr-AKH, which is released into the haemolymph, may have access to Rhopr-AKHR in the CNS, modifying behaviour associated with energy demands. Rhopr-AKHR expression was found in immature and adult reproductive tissues. The receptor transcript was highly expressed in the adult testes and female reproductive tissues, which include the bursa, oviducts, spermatheca and cement gland. Although a role for $\mathrm{AKH}$ in reproduction in insects has not yet been established, these data point towards additional functional conservation between $\mathrm{AKH}$ and $\mathrm{GnRH}$. The presence of the receptor transcript in female reproductive tissues indicates a possible role for Rhopr-AKH in egg production 
and/or egg-laying behaviour as has been shown in G. bimaculatus (Lorenz, 2003) and C. elegans (Lindemans et al., 2009), respectively. The expression in testes is intriguing, although not unique to $R$. prolixus. AKHR has also been shown to be expressed in $B$. mori testes (Yamanaka et al., 2008). There is no clear indication of the role that $\mathrm{AKH}$ may play in this tissue. It may, of course, have something to do with energy requirements during growth and development. Clearly, future experiments are required to examine these possibilities.

\section{Experimental procedures}

Animals

Unfed fifth-instar and adult $R$. prolixus were taken from a longstanding colony at the University of Toronto Mississauga. Insects were raised in incubators maintained at $60 \%$ humidity and $25{ }^{\circ} \mathrm{C}$, and were artificially fed on defibrinated rabbit blood (Hemostat Laboratories, Dixon, CA, USA; supplied by Cedarlane Laboratories Inc., Burlington, ON, Canada).

Isolation of $C D N A$ sequences encoding $\mathrm{R}$. prolixus $A K H$ and its receptor

Supercontig sequences representing the $R$.prolixus genome assembly (January 2012 release) were downloaded from VectorBase (https://www.vectorbase.org/) and imported into GENEIOUS 4.7.6 in order to perform local BLAST searches.

The A. gambiae AKHR (AAQ63187.1) amino acid sequence was used to mine the $R$. prolixus genome for putative AKHR. Primers specific to the hit regions were designed (Table S1) and used to amplify the partial cDNA sequence encoding a putative Rhopr-AKHR. Plasmid DNA isolated from a fifth-instar $R$. prolixus CNS cDNA library was used as the template for PCR (Paluzzi et al., 2008). The complete cDNA sequence encoding RhoprAKHR was obtained using a modified $5^{\prime}$ and $3^{\prime}$ rapid amplification of CDNA ends (RACE) PCR approach described previously (Zandawala et al., 2011, 2012). Gene-specific primers for $5^{\prime}$ and $3^{\prime}$ RACE PCRs were designed using the partial Rhopr-AKHR sequence. For 5' RACE, two gene-specific reverse primers and two plasmid-specific forward primers were used (Table S2). For $3^{\prime}$ RACE, two gene-specific forward primers and two plasmidspecific reverse primers were used (Table S3). A nested PCR approach was used to amplify the specific products, which were cloned using pGEM-T Easy vector (Promega Corporation, Madison, WI, USA) and sequenced at the SickKids DNA Sequencing Facility (The Centre for Applied Genomics, Hospital for Sick Children, Toronto, ON, Canada). All the PCR reactions were performed using a s1000 thermal cycler (Bio-Rad Laboratories, Mississauga, ON, Canada) using the following temperature-cycling profile: initial denaturation at $95^{\circ} \mathrm{C}$ for $3 \mathrm{~min}$, followed by 39 cycles of $94^{\circ} \mathrm{C}$ for $30 \mathrm{~s}, 50-60{ }^{\circ} \mathrm{C}$ (depending on the primers used) for $30 \mathrm{~s}$ and $72{ }^{\circ} \mathrm{C}$ for $1 \mathrm{~min}$, followed by a final extension at $72{ }^{\circ} \mathrm{C}$ for $10 \mathrm{~min}$. Lastly, the complete cDNA sequence encoding Rhopr-AKHR was amplified with a highfidelity Taq polymerase using the primers listed in Table S4.

Previously, Ons et al. (2011) reported the Rhopr-AKH prepropeptide sequence. This sequence was used to perform a tBLASTn search. Primers were designed based on the hits
(Table S1) and used to verify the complete ORF of Rhopr-AKH. In order to ensure that there were no additional methionine residues upstream of this sequence, and thus rule out the possibility of a larger ORF, 5' RACE PCRs were performed with the primers listed in Table S2. The largest CDNA fragment for this gene was amplified with a high-fidelity Taq polymerase using the primers listed in Table S4. The verification and extension of this sequence were necessary in order to design suitable primers for qPCR (see below).

\section{Sequence and phylogenetic analysis}

The intron-exon boundaries of Rhopr-AKH (submitted to GenBank under accession number KM283242) and RhoprAKHR (submitted to GenBank under accession number KF534791) were predicted using BLAST and confirmed with a splice site prediction software (Reese et al., 1997). The potential signal peptide cleavage site and dibasic cleavage site in the Rhopr-AKH prepropeptide were predicted using the SignalP 4.1 (Petersen et al., 2011) and ProP 1.0 (Duckert et al., 2004) servers, respectively. With regards to Rhopr-AKHR, the membrane topology was predicted using the TMHMM server v. 2.0 (Krogh et al., 2001), whereas potential intracellular phosphorylation sites and extracellular $\mathrm{N}$-linked glycosylation sites were predicted using the NetPhos 2.0 (Blom et al., 1999) and NetNGlyc 1.0 servers (http://www.cbs.dtu.dk/services/ NetNGlyc/ - last accessed on 22 September 2014), respectively. GRIFFIN (G protein and receptor interaction feature finding system; Yabuki et al., 2005) and PRED-COUPLE 2.0 (Sgourakis et al., 2005) were used to predict the coupling selectivity of Rhopr-AKHR to different G-proteins.

Rhopr-AKH prepropeptide was aligned with its orthologues from Nilaparvata lugens (BAO00932.1), D. melanogaster (NP_523918.1), Tribolium castaneum (ABB58739.1) and An. gambiae (ABD43194.1) using Clustal Omega (http://www.ebi .ac.uk/Tools/msa/clustalo/ - last accessed on 22 September 2014). Rhopr-AKHR was aligned with its orthologues from T. castaneum (NP_001076809), An. gambiae (AAQ63187.1), Apis mellifera (NP_001035354.1), C. elegans (NP_001249720.1) and H. sapiens (NP_000397.1). Phylogenetic relationships between various $\mathrm{AKH}$ and $\mathrm{GnRH}$ receptors were inferred using neighbour-joining analysis in MEGA6 (Tamura et al., 2013). Bootstrap values are based on 1000 iterations. All the sequences used for phylogenetic analysis are presented in Appendix S1.

\section{Spatial expression analyses using $q P C R$}

Various reports indicate that in most insects, AKH is expressed solely in the endocrine cells of the CC (see Schooley et al., 2012). To confirm that this is the case in R. prolixus, Rhopr-AKH expression was examined in the following tissues: fifth-instar CNS, fifthinstar CC/CA complex, adult CNS and the adult CC/CA complex. CNS samples included the brain (minus the CC/CA complex) and all of the ganglion. Rhopr-AKHR expression was also examined in these same tissues. To identify the target tissues of Rhopr$\mathrm{AKH}$, Rhopr-AKHR expression was examined in various tissues from fifth-instar $R$. prolixus and reproductive tissues from adult $R$. prolixus. The following tissues were dissected from fifthinstars: CNS (including the CC/CA complex), dorsal vessel, pool of fat bodies, diaphragm and abdominal nerves, foregut, salivary glands, anterior midgut, posterior midgut, Malpighian tubules, 
hindgut, male reproductive tissues, female reproductive tissues and PGs with its associated fat bodies. The following tissues were dissected from adults: testes, rest of the male reproductive tissues, ovaries and rest of the female reproductive tissues. Methods described previously were used to perform qPCR (Zandawala et al., 2013). Briefly, total RNA was extracted from the above tissues using a PureLink RNA Mini Kit (Life Technologies Corporation, Carlsbad, CA, USA), which was followed by cDNA synthesis with iScript Reverse Transcription Supermix for RT-qPCR (Bio-Rad Laboratories Ltd). The resulting cDNA was diluted 10 -fold and used as template for qPCR. Target genes (Rhopr-AKH and Rhopr-AKHR) and three housekeeping genes (alpha-tubulin, beta-actin and ribosomal protein 49) (Paluzzi \& O'Donnell, 2012) were amplified using the primers listed in Table S5 and their expression levels were determined using the delta-delta threshold-cycle method. A no-template negative control was also included for each experiment. Expression levels of Rhopr-AKH and Rhopr-AKHR were normalized via the geometric averaging of the transcript levels of the three housekeeping genes. At least two biological replicates (see each figure caption for the exact number) for each experiment and two technical replicates per reaction were used.

\section{$R N A i$}

Rhopr-AKHR (845 bp) and ARG (575 bp) fragments were amplified via PCR using the primers listed in Table S6. Plasmids containing the full-length Rhopr-AKHR and pGEM-T Easy vector (Promega, Madison, WI, USA) were used as the respective templates for these PCRs. T7 promoter sequence was added to these fragments via PCR using the primers listed in Table S6. The following temperature-cycling profile was used for all PCRs: initial denaturation at $94^{\circ} \mathrm{C}$ for $3 \mathrm{~min}$, followed by seven cycles of $94^{\circ} \mathrm{C}$ for $30 \mathrm{~s}, 58^{\circ} \mathrm{C}$ for $30 \mathrm{~s}$ and $72{ }^{\circ} \mathrm{C}$ for $90 \mathrm{~s}, 30$ cycles of $94{ }^{\circ} \mathrm{C}$ for $30 \mathrm{~s}, 62{ }^{\circ} \mathrm{C}$ for $30 \mathrm{~s}$ and $72{ }^{\circ} \mathrm{C}$ for $90 \mathrm{~s}$, and a final extension at $72{ }^{\circ} \mathrm{C}$ for $10 \mathrm{~min}$. The final PCR product was purified and used as template to synthesize dsRNA with T7 Ribomax Express RNAi System (Promega). The manufacturer-supplied protocol was followed to synthesize and purify dsRNA, which was stored at $-80{ }^{\circ} \mathrm{C}$ until injection.

Male adult $R$. prolixus (10 days postfed as adults) were anaesthetized by briefly exposing them to $\mathrm{CO}_{2}$. Hamilton syringes were used to inject $1 \mu \mathrm{l}$ of $2 \mu \mathrm{g} / \mu \mathrm{l}$ Rhopr-AKHR dsRNA. Two sets of controls were utilized in this experiment. One group was injected with $1 \mu \mathrm{l}$ of $2 \mu \mathrm{g} / \mu \mathrm{l}$ ARG dsRNA whereas the other group was left untreated. Following injections, insects were allowed to recover for about $30 \mathrm{~min}$ and kept in an incubator at $28^{\circ} \mathrm{C}$ on a $16 \mathrm{~h}: 8 \mathrm{~h}$ light/dark cycle. Lipid assays and qPCR to determine knockdown efficiency were performed 4 days post-injection (or 14 days postfeeding). Knockdown efficiency in the ventral abdominal fat body sheets was determined using QPCR as described above.

\section{Fat body lipid content}

The fat body sheet lining the ventral abdominal segments was removed under physiological saline $(150 \mathrm{mM} \mathrm{NaCl}, 8.6 \mathrm{mM} \mathrm{KCl}$, $2 \mathrm{mM} \mathrm{CaCl}_{2}, 4 \mathrm{mM} \mathrm{NaHCO}_{3}$ and $8.5 \mathrm{mM} \mathrm{MgCl}_{2}, 5 \mathrm{mM}$ HEPES, $\mathrm{pH}$ 7.0), placed in $500 \mu \mathrm{l}$ isopropanol, and then sonicated and centrifuged. A $50 \mu \mathrm{l}$ sample was removed from the supernatant, placed in $400 \mu$ isopropanol and the lipid content was measured as previously described (Patel et al., 2014).

\section{Haemolymph lipid levels}

The haemolymph lipid content was measured in a $5 \mu \mathrm{l}$ sample withdrawn from the clipped wing buds using a marked glass capillary (Drummond Scientific Company, Broomall, PA, USA). The lipoprotein in the haemolymph was precipitated in $10 \%$ trichloroacetic acid (TCA; Sigma, Oakville, ON, Canada), centrifuged and the resulting pellet was processed to measure lipid levels as previously described (Patel et al., 2014).

\section{AKH lipid mobilization assay}

Insects were immobilized by a short exposure to $\mathrm{CO}_{2}$ and then injected with either $2 \mu \mathrm{l}$ of $5 \mathrm{pmol}$ of Rhopr-AKH (pQLTFSTDWamide) or saline (control). These injections were made between the meso- and metathoracic segments midventrally using a $10 \mu \mathrm{l}$ Hamilton syringe. Rhopr-AKH was purchased from Genscript Laboratories (Piscataway, NJ, USA), dissolved in 50\% CHROMASOLV (Sigma) and then stored at $-20^{\circ} \mathrm{C}$ in $10 \mu \mathrm{l}$ of $10^{-3} \mathrm{M}$ aliquots. The insects were left for $90 \mathrm{~min}$ and then a $5 \mu \mathrm{l}$ haemolymph sample was removed from the clipped wing base using a marked glass capillary (Drummond Scientific Company). Care was taken to ensure that no fat body droplets were withdrawn with the haemolymph. The haemolymph was then placed in $50 \mu \mathrm{l}$ of $10 \%$ TCA to precipitate the lipoprotein. All samples were then centrifuged for $10 \mathrm{~min}$ at $8800 \mathrm{~g}$ using an Eppendorf Centrifuge 4513 (Mississauga, ON, Canada). The pellets were then processed to determine the lipid levels as previously described (Patel et al., 2014). To ensure that the vehicle used to solubilize Rhopr-AKH had no effect on haemolymph lipid levels, controls were run in which insects were injected with appropriately diluted CHROMASOLV. These insects did not show any change in haemolymph lipid levels, indicating that CHROMASOLV did not interfere with this experiment.

\section{Statistical analysis}

Graphical representations and statistical analysis were performed using GraphPad Prism (Version 5) (http://www.graphpad.com).

\section{Acknowledgements}

The authors wish to thank Nikki Sarkar for maintaining the colony and Himali Patel for technical support. This work was supported by Natural Sciences and Engineering Research Council of Canada (NSERC) Discovery Grants to I. O. and A. B. L., and a NSERC Canadian Graduate Scholarship to M. Z.

\section{References}

Attardo, G.M., Benoit, J.B., Michalkova, V., Yang, G., Roller, L., Bohova, J. et al. (2012) Analysis of lipolysis underlying lactation in the tsetse fly, Glossina morsitans. Insect Biochem Mol Biol 42: 360-370.

Audsley, N., Down, R.E. and Isaac, R.E. (2014) Genomic and peptidomic analyses of the neuropeptides from the emerging pest, Drosophila suzukii. Peptides (in press).

Bednarova, A., Kodrik, D. and Krishnan, N. (2013) Adipokinetic hormone exerts its anti-oxidative effects using a conserved 
signal-transduction mechanism involving both PKC and cAMP by mobilizing extra- and intracellular $\mathrm{Ca} 2+$ stores. Comp Biochem Physiol C Toxicol Pharmacol 158: 142-149.

Blom, N., Gammeltoft, S. and Brunak, S. (1999) Sequence and structure-based prediction of eukaryotic protein phosphorylation sites. J Mol Biol 294: 1351-1362.

Broomfield, C.E. and Hardy, P.M. (1977) The synthesis of locust adipokinetic hormone. Tetrahedron Lett 18: 2201-2204.

Carlisle, J. and Loughton, B. (1986) The inhibition of protein synthesis in Locusta migratoria by adipokinetic hormone. $J$ Insect Physiol 32: 573-578.

Duckert, P., Brunak, S. and Blom, N. (2004) Prediction of proprotein convertase cleavage sites. Protein Eng Des Sel17: 107-112.

Gäde, G. and Marco, H.G. (2013) AKH/RPCH peptides. In Handbook of Biologically Active Peptides (A. Kastin ed.), pp. 185190. Academic Press, San Diego, CA.

Grimmelikhuijzen, C.J. and Hauser, F. (2012) Mini-review: the evolution of neuropeptide signaling. Regul Pept 177 (Suppl.): S6-S9.

Hansen, K.K., Hauser, F., Cazzamali, G., Williamson, M. and Grimmelikhuijzen, C.J. (2006) Cloning and characterization of the adipokinetic hormone receptor from the cockroach Periplaneta americana. Biochem Biophys Res Commun 343: 638-643.

Hansen, K.K., Stafflinger, E., Schneider, M., Hauser, F., Cazzamali, G., Williamson, M. et al. (2010) Discovery of a novel insect neuropeptide signaling system closely related to the insect adipokinetic hormone and corazonin hormonal systems. J Biol Chem 285: 10736-10747.

Hauser, F. and Grimmelikhuijzen, C.J. (2014) Evolution of the $\mathrm{AKH} /$ corazonin/ACP/GnRH receptor superfamily and their ligands in the Protostomia. Gen Comp Endocrinol 209: 35-49.

Isabel, G., Martin, J.R., Chidami, S., Veenstra, J.A. and Rosay, P. (2005) AKH-producing neuroendocrine cell ablation decreases trehalose and induces behavioral changes in Drosophila. Am J Physiol Regul Integr Comp Physiol 288: R531-R538.

Kaufmann, C. and Brown, M.R. (2006) Adipokinetic hormones in the African malaria mosquito, Anopheles gambiae: identification and expression of genes for two peptides and a putative receptor. Insect Biochem Mol Biol 36: 466-481.

Kaufmann, C., Merzendorfer, H. and Gade, G. (2009) The adipokinetic hormone system in Culicinae (Diptera: Culicidae): molecular identification and characterization of two adipokinetic hormone (AKH) precursors from Aedes aegypti and Culex pipiens and two putative $\mathrm{AKH}$ receptor variants from A. aegypti. Insect Biochem Mol Biol 39: 770-781.

Keeley, L., Hayes, T., Bradfield, J. and Sowa, S. (1991) Physiological actions by hypertrehalosemic hormone and adipokinetic peptides in adult Blaberus discoidalis cockroaches. Insect Biochem 21: 121-129.

Konuma, T., Morooka, N., Nagasawa, H. and Nagata, S. (2012) Knockdown of the adipokinetic hormone receptor increases feeding frequency in the two-spotted cricket Gryllus bimaculatus. Endocrinology 153: 3111-3122.

Krogh, A., Larsson, B., von Heijne, G. and Sonnhammer, E.L. (2001) Predicting transmembrane protein topology with a hidden Markov model: application to complete genomes. J Mol Biol 305: 567-580.
Lindemans, M., Liu, F., Janssen, T., Husson, S.J., Mertens, I., Gade, G. et al. (2009) Adipokinetic hormone signaling through the gonadotropin-releasing hormone receptor modulates egglaying in Caenorhabditis elegans. Proc Natl Acad Sci USA 106: $1642-1647$.

Lorenz, M.W. (2003) Adipokinetic hormone inhibits the formation of energy stores and egg production in the cricket Gryllus bimaculatus. Comp Biochem Physiol B Biochem Mol Biol 136: 197-206.

Lorenz, M.W. and Anand, A.N. (2004) Changes in the biochemical composition of fat body stores during adult development of female crickets, Gryllus bimaculatus. Arch Insect Biochem Physiol 56: 110-119.

Malik, A., Gade, G. and Lange, A.B. (2012) Sequencing and biological effects of an adipokinetic/hypertrehalosemic peptide in the stick insect, Baculum extradentatum. Peptides 34: 51-56.

Marco, H.G., Simek, P., Clark, K.D. and Gade, G. (2013) Novel adipokinetic hormones in the kissing bugs Rhodnius prolixus, Triatoma infestans, Dipetalogaster maxima and Panstrongylus megistus. Peptides 41: 21-30.

Millar, R.P., Lu, Z.L., Pawson, A.J., Flanagan, C.A., Morgan, K. and Maudsley, S.R. (2004) Gonadotropin-releasing hormone receptors. Endocr Rev 25: 235-275.

Noyes, B.E., Katz, F.N. and Schaffer, M.H. (1995) Identification and expression of the Drosophila adipokinetic hormone gene. Mol Cell Endocrinol 109: 133-141.

Ons, S., Sterkel, M., Diambra, L., Urlaub, H. and Rivera-Pomar, R. (2011) Neuropeptide precursor gene discovery in the Chagas disease vector Rhodnius prolixus. Insect Mol Biol 20: 29-44.

Paluzzi, J.P. and O'Donnell, M.J. (2012) Identification, spatial expression analysis and functional characterization of a pyrokinin-1 receptor in the Chagas' disease vector, Rhodnius prolixus. Mol Cell Endocrinol 363: 36-45.

Paluzzi, J.P., Russell, W.K., Nachman, R.J. and Orchard, I. (2008) Isolation, cloning, and expression mapping of a gene encoding an antidiuretic hormone and other CAPA-related peptides in the disease vector, Rhodnius prolixus. Endocrinology 149: 4638-4646.

Park, Y., Kim, Y.J. and Adams, M.E. (2002) Identification of G protein-coupled receptors for Drosophila PRXamide peptides, CCAP, corazonin, and AKH supports a theory of ligandreceptor coevolution. Proc Natl Acad Sci USA 99: 1142311428.

Patel, H., Orchard, I., Veenstra, J.A. and Lange, A.B. (2014) The distribution and physiological effects of three evolutionarily and sequence-related neuropeptides in Rhodnius prolixus: adipokinetic hormone, corazonin and adipokinetic hormone/corazonin-related peptide. Gen Comp Endocrinol 195: $1-8$.

Petersen, T.N., Brunak, S., von Heijne, G. and Nielsen, H. (2011) SignalP 4.0: discriminating signal peptides from transmembrane regions. Nat Methods 8: 785786.

Reese, M.G., Eeckman, F.H., Kulp, D. and Haussler, D. (1997) Improved splice site detection in Genie. J Comput Biol 4: 311-323.

Roch, G.J., Busby, E.R. and Sherwood, N.M. (2014) GnRH receptors and peptides: skating backward. Gen Comp Endocrinol 209: 118-134. 
Rosiński, G. and Gäde, G. (1988) Hyperglycaemic and myoactive factors in the corpora cardiaca of the mealworm, Tenebrio molitor. J Insect Physiol 34: 1035-1042.

Schooley, D.A., Horodyski, F.M. and Coast, G.M. (2012) Hormones controlling homeostasis in insects. In Insect Endocrinology (Gilbert, L.I., ed.), pp. 366-429. Academic Press, San Diego, CA.

Sgourakis, N.G., Bagos, P.G., Papasaikas, P.K. and Hamodrakas, S.J. (2005) A method for the prediction of GPCRs coupling specificity to G-proteins using refined profile Hidden Markov Models. BMC Bioinformatics 6: 104.

Siegert, K.J. (1999) Locust corpora cardiaca contain an inactive adipokinetic hormone. FEBS Lett 447: 237-240.

da Silva, S.R., da Silva, R. and Lange, A.B. (2011) Effects of crustacean cardioactive peptide on the hearts of two Orthopteran insects, and the demonstration of a Frank-Starling-like effect. Gen Comp Endocrinol 171: 218-224.

Staubli, F., Jorgensen, T.J., Cazzamali, G., Williamson, M., Lenz, C., Sondergaard, L. et al. (2002) Molecular identification of the insect adipokinetic hormone receptors. Proc Natl Acad Sci USA 99: 3446-3451.

Stone, J.V., Mordue, W., Batley, K.E. and Morris, H.R. (1976) Structure of locust adipokinetic hormone, a neurohormone that regulates lipid utilisation during flight. Nature 263: 207-211.

Tamura, K., Stecher, G., Peterson, D., Filipski, A. and Kumar, S. (2013) MEGA6: molecular evolutionary genetics analysis version 6.0. Mol Biol Evol 30: 2725-2729.

Ward, J.P., Candy, D.J. and Smith, S.N. (1982) Lipid storage and changes during flight by triatomine bugs (Rhodnius prolixus and Triatoma infestans). J Insect Physiol 28: 527-534.

Wicher, D., Agricola, H.J., Sohler, S., Gundel, M., Heinemann, S.H., Wollweber, L. et al. (2006) Differential receptor activation by cockroach adipokinetic hormones produces differential effects on ion currents, neuronal activity, and locomotion. J Neurophysiol 95: 2314-2325.

Yabuki, Y., Muramatsu, T., Hirokawa, T., Mukai, H. and Suwa, M. (2005) GRIFFIN: a system for predicting GPCR-G-protein coupling selectivity using a support vector machine and a hidden Markov model. Nucleic Acids Res 33: W148-W153.
Yamanaka, N., Yamamoto, S., Zitnan, D., Watanabe, K., Kawada, T., Satake, H. et al. (2008) Neuropeptide receptor transcriptome reveals unidentified neuroendocrine pathways. PLOS ONE 3: e3048.

Zandawala, M., Paluzzi, J.P. and Orchard, I. (2011) Isolation and characterization of the cDNA encoding $\mathrm{DH}(31)$ in the kissing bug, Rhodnius prolixus. Mol Cell Endocrinol 331: 79-88.

Zandawala, M., Lytvyn, Y., Taiakina, D. and Orchard, I. (2012) Cloning of the cDNA, localization, and physiological effects of FGLamide-related allatostatins in the bloodgorging bug, Rhodnius prolixus. Insect Biochem Mol Biol 42: 10-21.

Zandawala, M., Li, S., Hauser, F., Grimmelikhuijzen, C.J. and Orchard, I. (2013) Isolation and functional characterization of calcitonin-like diuretic hormone receptors in Rhodnius prolixus. PLoS ONE 8: e82466.

Ziegler, R., Isoe, J., Moore, W., Riehle, M.A. and Wells, M.A. (2011) The putative AKH receptor of the tobacco hornworm, Manduca sexta, and its expression. J Insect Sci 11: 40.

\section{Supporting Information}

Additional Supporting Information may be found in the online version of this article at the publisher's web-site:

Table S1. Primers used to amplify the complete ORF of Rhopr-AKH and partial cDNA sequence of Rhopr-AKHR.

Table S2. Primers used to perform 5' RACE PCR reactions.

Table S3. Primers used to perform 3' RACE PCR reactions.

Table S4. Primers used to amplify the largest cDNA fragments.

Table S5. Primers used for qPCR reactions.

Table S6. Primers used to generate Rhopr-AKHR and ampicillin resistance gene (ARG) dsRNA. (Note: The T7 promoter sequence is bolded).

Appendix S1. Sequences used for phylogenetic analysis. 